\title{
Reservoir Gate Based on Spiral Design
}

\author{
Guocai Chen ${ }^{\mathrm{a}}$, Jinxin Liu ${ }^{\mathrm{b}}$, Shuhao Zhang ${ }^{\mathrm{c}}$, Zhaonan $\mathrm{Li}^{\mathrm{d}}$ and Zhiyuan $\mathrm{Xu}^{\mathrm{e}}$, * \\ School of Navigation and Naval Architecture, Dalian Ocean University, Dalian 116000, China \\ a1634860344@qq.com, ba785789772@163.com, ‘476449706@qq.com, d739557278@qq.com, exuzhiyuan@ \\ dlou.edu.cn \\ *Corresponding author: xuzhiyuan@dlou.edu.cn
}

Keywords: new spiral, Gate, the river

\begin{abstract}
Objective] aiming at the existing gate structure problem, a new type of spiral gate was designed. [Design] new spiral gate relates to the technical field of hydraulic engineering, including: support, driving device and gate body; The support is fixed on the dam body, and the driving device is fixed on the support body, which is used to drive the gate body to rotate, and at the same time, the gate body moves along the thread on the gate slot. [Results] the problem of poor sealing between gate gate and gate slot was overcome, and the sealing effect between gate and dam was improved. [Conclusion] the designed structure can provide some reference for solving the problems of existing gate structure.
\end{abstract}

\section{Preface}

Gates are control facilities used to close and open drain (discharge) water channels, and are an important part of hydraulic structures. They are usually used to intercept water flows, control water levels, regulate flow, discharge sediment and floats, etc. It plays a key role in water conservancy projects, which are usually built on rivers and canals to control the flow and adjust the water level of low-head hydraulic structures. Closing the gate can block floods, tide or raise the upstream water level to meet irrigation, power generation, and shipping , Aquatic products, environmental protection, industrial and domestic water needs; opening the gate can release flood, waterlogging, abandoned water or waste water, and can also supply water to downstream rivers or channels. In water conservancy projects, the sluice acts as a stop, drain or take water Building, widely used.

But the existing problems of existing water gates are:

(1) There are problems such as poor sealing, laborious opening and closing, and poor flexibility at the gate connection. Furthermore, the opening and closing frequently causes eraser wear, or the possibility of submerging the bottom sill, which affects the function of retaining water and storing water. It is easy to carbonize and fall off, causing leakage in the deformation of the gate, greatly reducing the water storage capacity, and even greater accidents [1].

(2) Most of the existing gates are flat gates [2]. Due to the influence of water flow, gaps between the flat gates and the door grooves are prone to occur during use, resulting in a decrease in the tightness of the gates. Both the opening and closing processes require greater traction.

Because it is found that the spiral structure can effectively control the dredging and sealing of the water flow, and the structure is simple, it can not only save materials, but also reduce the use of 
electricity. Because it is a new structure, it remains to be studied in the subsequent use.

\section{Working principle and structural characteristics of new spiral gate}

\subsection{The working principle of the new spiral gate}

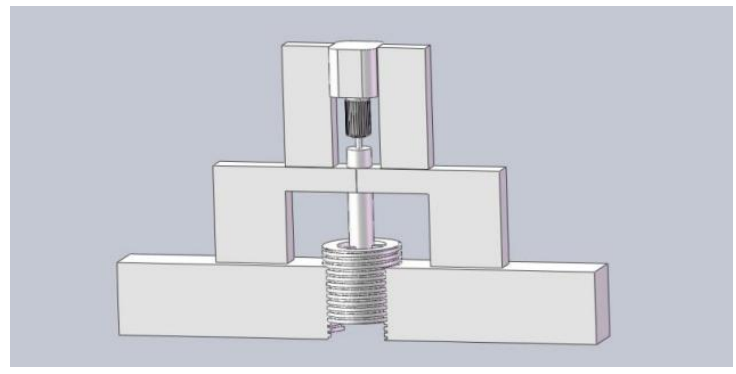

Figure 1. Structural drawing

The purpose of the new spiral gate is to solve the problems existing in the prior art mentioned above. The new spiral gate can not only improve the seal of the gate, but also reduce the traction required when opening and closing the gate, and overcome the disadvantages of wear and tear. Structural innovation has been achieved to achieve ecological simplicity and efficiency. The design basis of any product must first be clear, understand its use, functional requirements, use environment, etc., and then enter the next step of design [3].

\subsection{Structural Features of New Spiral Gate}

The new spiral gate provides a spiral gate including a bracket, a driving device and a gate; the bracket is fixed on the dam body, the driving device is fixed on the bracket, the side wall of the gate is provided with threads, the gate and the dam body The gate slot is threaded, and the output end of the driving device is connected to the gate for driving the gate to rotate. At the same time, the gate moves along the threads on the gate slot to open and close the threaded gate. The new spiral gate also includes a rotating shaft, a rotating shaft one end is fixedly connected to the output end of the driving device, and the other end of the rotating shaft is fixedly connected to the upper end of the gate.

The driving device includes a motor and a reducer. The motor is fixed on the bracket. The output of the motor is connected to the input of the reducer. The output of the reducer is connected to the upper end of the shaft. The bracket includes an upper bracket and a lower bracket. The ends are fixedly connected to the dam on both sides of the gate groove, the upper bracket is fixedly connected to the lower bracket, and the driving device is fixedly connected to the upper bracket. The upper end of the lower bracket is provided with a shaft hole for penetrating the shaft. The gate is cylindrical Hollow gate body. The new type spiral gate provides a spiral gate. By using a threaded connection between the gate body and the gate slot on the dam body, this arrangement can improve the seal between the gate body and the gate slot. 


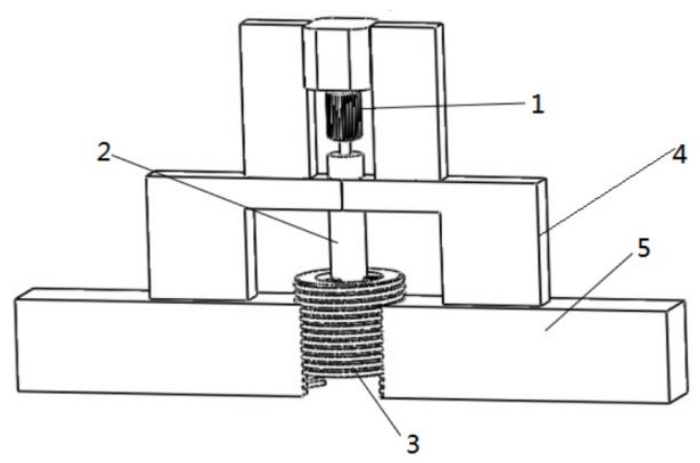

Figure 2. Three-dimensional map

The picture shows the spiral sluice structure

1-drive unit, 2-rotary shaft, 3-gate body, 4-bracket, 5-dam body.

The gate of the new spiral gate is threaded to the gate slot. Compared with the existing plate gate and the plate gate slot, it is beneficial to improve the seal of the gate, and the process of opening and closing the gate is driven by the drive device. The slanting motion is performed along the threads on the gate groove. Compared with the existing plate gate that moves in the vertical direction when it is opened and closed, the new spiral gate requires less traction during the opening and closing process.

The spiral gate also includes a rotating shaft, one end of the rotating shaft is fixedly connected to the output end of the driving device, and the other end of the rotating shaft is fixedly connected to the upper end of the gate body. The driving device includes a motor and a reducer, and the motor is fixedly arranged on the bracket, and the output end of the motor it is connected to the input of the reducer, and the output of the reducer is connected to the upper end of the shaft. The bracket includes an upper bracket and a lower bracket; the two ends of the lower bracket are fixedly connected to the dam on both sides of the gate slot, and the upper bracket is fixedly connected to on the lower bracket, the driving device is fixedly connected to the upper bracket. The upper end surface of the lower bracket is provided with a shaft hole for penetrating the shaft to limit the shaft. The gate body is a cylindrical hollow gate body, which saves material. It also saves effort in the process of opening and closing the gate.

\subsection{Data of the new spiral gate}

Gate size (height, outer diameter, inner diameter) $=5 \mathrm{~m} \mathrm{3m} \mathrm{2m}$

Dam size (height and thickness) $=4.8 \mathrm{~m}$ and $2.5 \mathrm{~m}$

Stand size (height, top width, bottom width, thickness) $=4 \mathrm{~m}, 14 \mathrm{~m}, 3.5 \mathrm{~m}, 1.5 \mathrm{~m}$

Shaft size (height and diameter) $=11.5 \mathrm{~m} 2 \mathrm{~m}$

Gate maximum water level: $4 \mathrm{~m}$

Maximum water blocking height: $4.65 \mathrm{~m}$

Maximum submerged water depth: $2.62 \mathrm{~m}$

Gate total water pressure: $10539.35 \mathrm{kN}$

Operation mode: Still water opening and closing

Hoist type: direct-connected hydraulic hoist

Hoist capacity: $3200 \mathrm{kN}$

The above parameters are virtual parameters designed by us, and can be scaled up or down in the same proportion as needed based on the requirements. The specific parameters are subject to actual conditions. 


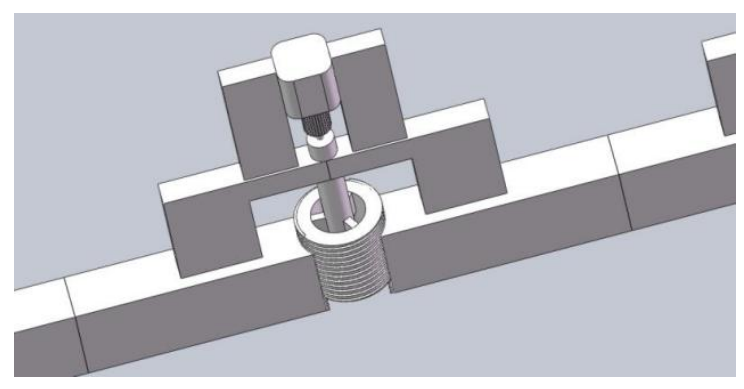

Figure 3. Stereograph

\section{Gate force analysis}

\subsection{Stress analysis}

The pressure of the sluice in the river is very large. Among them, the factors that affect the pressure of the sluice are mainly related to the descent depth and the river water density. The greater the density of the river water, the greater the pressure on the sluice. The greater the depth of descent, the greater the pressure on the gate. Combining the analysis of the relevant data of the semicircular arc tunnel, the calculation formula for the pressure load received by the spiral gate and basic assumption of flow field in a literature is [4]:

$\mathrm{P}=0.4 \times 2^{s-1} \mu \omega, \omega=1+\mathrm{i}(\mathrm{B}-5)$

Where $\mathrm{P}$ is the vertical uniformly distributed surrounding rock pressure, $\mathrm{kPa}$; $\mathrm{S}$ is the surrounding rock level; $\mu$ is the natural gravity of the surrounding rock, $\mathrm{kN} / \mathrm{m} 3 ; \omega$ is the width influence factor; $\mathrm{B}$ is the excavation width of the spiral gate, $\mathrm{m}$; $\mathrm{i}$ It is the rate of increase and decrease of water pressure, and the corresponding value is taken with $\mathrm{B}$ as the standard. When $\mathrm{B}$ exceeds $5 \mathrm{~m}, \mathrm{i}=0.1$; when $\mathrm{B}$ is less than $5 \mathrm{~m}, \mathrm{i}=0.2$.

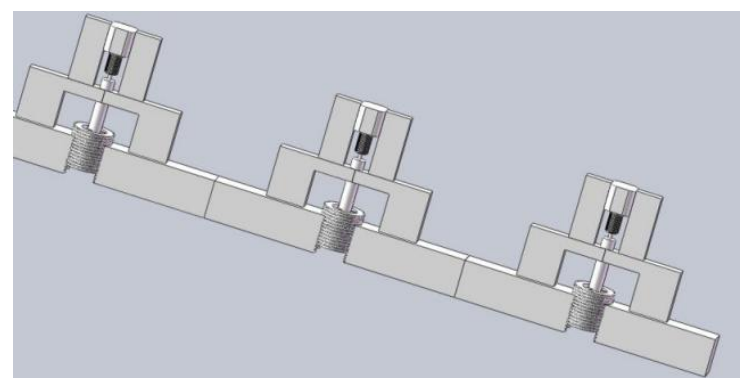

Figure 4. Simulation Diagram

\subsection{Friction analysis}

Since the spiral sluice is usually located in a freshwater channel, for common liquids, it meets Newton's law of internal friction. The friction of the water film $\mathrm{f}$ is related to the dynamic viscosity $\mu$ and velocity gradient of the liquid, the formula is obtained after drawing lessons from the numerical simulation method of some literature [5]:

$$
\mathrm{F}=\int_{R_{1}}^{R_{0}} \mu \frac{\partial v}{\partial \delta} \bullet 2 \pi d r
$$




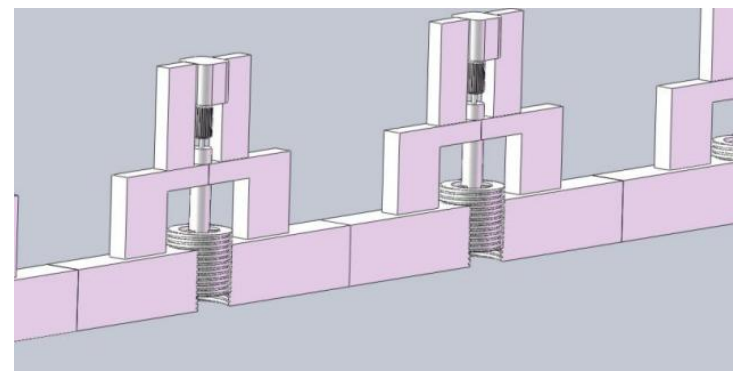

Figure 5. Sketch map

\section{Conclusion}

The new spiral gate draws inspiration from the spiral bottle cap, adopts the concentric spiral cylinder rotation method, the bottle cap formula and arc design. The screw of the cylinder is driven by the upper motor to drive the sluice to rise and fall, which overcomes the existing The defects of poor sealing between the flat gate and the door slot and the large traction force required to open and close the flat gate can improve the tightness between the gate and the dam and reduce the traction required when opening and closing. When the gate is rotated to the bottom of the dam, it is tightly closed to achieve the purpose of sealing.

At present, the construction of sluice gates is developing in the direction of diversified forms, lighter structure, construction assembly, operation automation and remote control. The new spiral gate is simple in structure and short in production time after the design is completed. The materials for spiral gates are widely selected the required manufacturing technology is simple, easy to manufacture, more secure, and has a longer service life.

\section{Acknowledgments}

This work was financially supported by Innovation and entrepreneurship training program for college students in Dalian Ocean University in 2019 fund (201910158030).

\section{References}

[1] Liu Xuhui, Zhang Qing, Xie Lihua, et al. A fast and precise guidance and sealed flood gate: zl201410168255.9 [p] .2016-01-06.

[2] Zhang Qing, Liu Xuhui, Xie Lihua's new helicopter flood control gate and its application: 1008 -536x (2016) 04-0035-03

[3] Pan Changyong A design of Spiral Elevator: 1002-2333 (2018) 11-0057-04

[4] Ding Xuexing, Fu Yingjie, Zhang Jing, et al. Flow pattern analysis of flow field of spiral groove dry gas seal end face based on CFD [j]. Journal of Drainage and Irrigation Machinery Engin, 2010, 28 (4): 330-334.

[5] Ding Xuexing, Xu Jie, Zhang Weizheng, Lu Junjie. The law of friction coefficients of spiral groove dry gas seal lubrication gas film. 2019, 32 (02), 84-91 